Research report

\title{
Pronociception from the dorsomedial nucleus of the hypothalamus is mediated by the rostral ventromedial medulla in healthy controls but is absent in arthritic animals
}

\author{
Filipa Pinto-Ribeiro ${ }^{\mathrm{a}, \mathrm{b}, 1}$, Diana Amorim ${ }^{\mathrm{a}, \mathrm{b}, 1}$, Ana David-Pereira ${ }^{\mathrm{a}}$, Ana Maria Monteiro ${ }^{\mathrm{a}, \mathrm{c}}$, \\ Patricio Costa ${ }^{\mathrm{a}, \mathrm{b}}$, Antti Pertovaara ${ }^{\mathrm{d}}$, Armando Almeida $^{\mathrm{a}, \mathrm{b}, *}$ \\ a Life and Health Sciences Research Institute (ICVS), School of Health Sciences (ECS), Campus of Gualtar, University of Minho, $4750-057$ Braga, Portugal \\ b ICVS/3B's - PT Government Associate Laboratory, Braga/Guimarães, Portugal

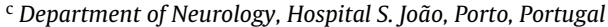 \\ ${ }^{\mathrm{d}}$ Biomedicum Helsinki, Institute of Biomedicine/Physiology, University of Helsinki, POB 63, Helsinki, Finland
}

\section{A R T I C L E I N F O}

\section{Article history:}

Received 16 April 2013

Received in revised form

27 September 2013

Accepted 1 October 2013

Available online $\mathrm{xxx}$

\section{Keywords:}

Dorsomedial hypothalamic nucleus (DMH)

Arthritic pain

Descending pain modulation

Rostral ventromedial medulla (RVM)

Pronociception

ON-like cells

\begin{abstract}
A B S T R A C T
The dorsomedial nucleus of the hypothalamus $(\mathrm{DMH})$ has been proposed to participate in stress-induced hyperalgesia through facilitation of pronociceptive cells in the rostroventromedial medulla (RVM). We hypothesized that the DMH participates in hyperalgesia induced by arthritis. The DMH was pharmacologically manipulated while assessing heat-evoked nociceptive behavior or the discharge rates of pronociceptive RVM ON- and antinociceptive RVM OFF-like cells in NAIVE, SHAM and monoarthritic (ARTH) animals. In NAIVE and SHAM animals, the changes in nociceptive behavior induced by activation of the DMH by glutamate and inhibition by lidocaine were in line with earlier evidence indicating that the DMH has a nociceptive facilitating role. However, in ARTH animals, neither activation nor inhibition of the DMH influenced pain-like behavior evoked by stimulation of an uninflamed skin region (paw and tail). In accordance with these behavioral results, activation or inhibition of the DMH induced pronociceptive changes in the discharge rates of RVM cells in NAIVE and SHAM animals, which suggests that the DMH has a pronociceptive role mediated by the RVM in normal animals. However, in ARTH animals, both glutamate and lidocaine in the $\mathrm{DMH}$ failed to influence either pain-like behavior or noxious stimulation-evoked responses of RVM cells, while blocking the DMH increased spontaneous activity in the pronociceptive RVM ON cells. Our data indicate that the DMH participates in descending facilitation of cutaneous nociception in healthy controls, but it is not engaged in the regulation of cutaneous nociception in monoarthritic animals, while a minor role in tonic suppression of nociception in arthritis cannot be discarded.
\end{abstract}

(c) 2013 Elsevier Inc. All rights reserved.

\section{Introduction}

It is widely accepted that central sensitization contributes to the development and maintenance of chronic pain (Schaible et al.,

Abbreviations: ARTH, arthritic animals/arthritis; CTRL, control animals/controls; $\mathrm{DMH}$, dorsomedial nucleus of the hypothalamus; GLU, glutamate; H, peripheral noxious heat stimulation; HPA, hypothalamus-pituitary-adrenal axis; LIDO, lidocaine; MS, mechanical stimulation; NPS, noxious peripheral stimulation; P, peripheral noxious mechanical stimulation; PAG, periaqueductal gray matter; PW, paw-withdrawal; RVM, rostral ventromedial medulla; SAL, saline solution; TF, tailflick; TS, thermal stimulation.

* Corresponding author at: Life and Health Sciences Research Institute (ICVS), School of Health Sciences (ECS), Campus of Gualtar, University of Minho, 4710-057 Braga, Portugal. Tel.: +351 253604808; fax: +351 253604809 .

E-mail address: aalmeida@ecsaude.uminho.pt (A. Almeida).

1 These authors contributed equally for this paper.
2002). Alterations of the descending pain control systems exert an important role in central sensitization (Latremoliere and Woolf 2009; Sandkühler, 2009). In chronic pain conditions, functional changes have been described both in descending facilitatory and inhibitory circuits (Herrero and Cervero, 1996; Gozariu et al., 1998; Burgess et al., 2002) that influence nociception at the spinal cord level (Ren and Dubner, 1996; Urban and Gebhart, 1999). Several studies have already demonstrated that pathways descending from the rostral ventromedial medulla (RVM) facilitate pain-related responses in some chronic pain conditions (Burgess et al., 2002; Ossipov et al., 2010). More specifically, it has been reported that changes in the activity of RVM cells are essential to the development of hyperalgesia (Ossipov et al., 2005). Nociception is enhanced when the basal and stimulus-evoked activities of pronociceptive RVM ON-cells are increased and those of antinociceptive OFF-like cells are decreased (Miki et al., 2002). In chronic pain models, the net effect by the RVM may be pronociception, since blockade 
of the RVM has reduced hyperalgesia (see for review, Urban and Gebhart, 1999). Interestingly, Urban and Gebhart (1999) reported that blocking the RVM reversed secondary hyperalgesia (i.e., hyperalgesia adjacent to the injured region) to heat in monoarthritic or mustard oil-treated animals but failed to reduce primary hyperalgesia (i.e., hyperalgesia in the injured region) in animals with paw inflammation. This result is in line with the hypothesis that the RVM-mediated descending modulation of spinal nociception may vary from inhibition to facilitation depending on whether the spinal neuron receives inputs from the inflamed/injured area or from adjacent areas, respectively (Vanegas, 2004; Vanegas and Schaible, 2004).

The dorsomedial nucleus of the hypothalamus (DMH) is among the supramedullary structures that have been shown to contribute to descending pain control through a relay in the RVM (Heinricher et al., 2009). In healthy rats, the DMH has a top-down pronociceptive action mediated by the RVM. This was shown by the finding that disinhibition of the $\mathrm{DMH}$ with the $\mathrm{GABA}_{\mathrm{A}}$ receptor antagonist bicuculline induced behavioral hyperalgesia that was accompanied by increased activation of pronociceptive RVM ON-cells and decreased activation of antinociceptive RVM OFF-cells (Martenson et al., 2009). However, it is still not known whether a chronic pain condition, such as arthritis, induces changes in the top-down pronociceptive action by the DMH. Therefore, we assessed whether the top-down effect by the DMH on the discharge rates of RVM cells and pain behavior differs between monoarthritic and healthy control animals. Since the DMH has been shown to promote nociception through a relay in the RVM in healthy controls (Martenson et al., 2009) and since the descending pain facilitation by the RVM in pathophysiological conditions has been most prominent outside of the injured region (Urban et al., 1999), pain behavior in the monoarthritic animals was assessed by stimulating the intact skin outside the dermatome corresponding to the inflamed joint (paw and tail).

We hypothesized that arthritis increases the descending pronociceptive action of the DMH and thereby contributes to chronic pain and hypersensitivity in arthritis. Results of the present study, however, were opposite to that predicted by our working hypothesis: assessment of pain behavior and the discharge rates of pro- and antinociceptive RVM cells indicated that the top-down pronociceptive action by the DMH was reduced in arthritis.

\section{Materials and methods}

\subsection{Animals, ethical issues and anesthesia}

The experiments were performed in adult male Wistar rats with 175-250 g (Charles River, Barcelona, Spain). The experimental protocol was approved by the Institutional Ethical Commission and followed the European Community Council Directive 86/609/EEC and 2010/63/EU concerning the use of animals for scientific purposes. All efforts were made to minimize animal suffering and to use only the number of animals necessary to produce reliable scientific data.

During surgery and electrophysiological sessions, the anesthesia was induced (50 mg/kg, i.p.) and maintained by administering pentobarbitone $(15-20 \mathrm{mg} / \mathrm{kg} / \mathrm{h}$, i.p.). After the initial anesthesia for the placement of the animal in the stereotaxic frame and for the placement of the injection cannula and the recording electrode, the animals received three additional reinforcements $(20 \mathrm{mg} / \mathrm{kg}$ ) at $60 \mathrm{~min}$ intervals or more frequently when needed (see below). The level of anesthesia was monitored by verifying dilation of the pupils, the general muscle tone and behavioral responses to noxious pinching every $15 \mathrm{~min}$. If the pinch-evoked response was stronger than a brief muscle twitch restricted to the stimulated limb, the animal was given an additional dose of pentobarbitone $(20 \mathrm{mg} / \mathrm{kg}$ i.p.) and the anesthesia level was re-assessed within $5 \mathrm{~min}$. After the completion of the behavioral tasks or the electrophysiological sessions, animals received a lethal dose of pentobarbitone and the brains were removed for histological confirmation of cannula placement (Fig. 1B-K) or electrode recording site.

\subsection{Induction of arthritis}

The induction of arthritis (ARTH) was performed 21 days before the actual experiments, as described in detail elsewhere (Ansah and Pertovaara, 2007). Briefly, a solution of 3\% kaolin and 3\% carrageenan (Sigma-Aldrich, St. Louis, MO, USA) was injected into the synovial cavity of the right knee joint at a volume of $0.1 \mathrm{~mL}$. This model produces mechanical hyperalgesia, which begins just in a few hours after surgery and extends up to 8 weeks (Radhakrishnan et al., 2003). In each animal, development of arthritis was verified $1-2 \mathrm{~h}$ prior to each experiment. Only those rats that vocalized every time after five flexion-extension movements of the knee joint were considered to have arthritis and were included in the arthritis group. SHAM animals were injected with $0.1 \mathrm{~mL}$ of saline in the synovial cavity of the left knee joint. NAIVE animals were not submitted to any type of treatments of the synovial cavity.

\subsection{Procedures for intracerebral microinjections}

For intracerebral drug administration, one (electrophysiology) or two (behavioral assessments) stainless steel guide cannulae (26 gauge; PlasticsOne, Roanoke, VA, USA) were implanted in the DMH and the RVM (behavioral assessments). Animals were allowed to recover from the surgery for at least one week before any tests were performed.

Test drugs were administered in either the DMH or the RVM through a 33-gauge injection cannula (PlasticsOne) with an injection volume of $0.5 \mu \mathrm{L}$. The spread of the injected drugs within the brain was expected to have a diameter of at least $1 \mathrm{~mm}$ (Myers, 1966). The efficacy of injections was monitored by watching the movement of a small air bubble through the tubing. The injection lasted $20 \mathrm{~s}$ and the injection cannula was left in place for additional $30 \mathrm{~s}$ to minimize the return of drug solution back to the injection cannula.

Additionally, for physiological confirmation of cannula placement in the $\mathrm{DMH}$ (disinhibiton of the $\mathrm{DMH}$ and nociceptive facilitation), an injection of bicuculline methiodide, a $G_{A B A}$ receptor antagonist ( $0.4 \mathrm{mM}$; Tocris Bioscience, Bristol, UK), was performed in the $\mathrm{DMH}$ in each animal before the beginning of the behavioral testing (Fig. 1A) as well as at the end of each electrophysiological session, in order to reproduce the earlier results by Martenson et al. (2009). Only the injections involving the DMH (Fig. 1B-G) resulted in decreased paw-withdrawal latency. No alterations were observed after injections outside the DMH area, or an antinociceptive effect was observed (i.e., ventromedial hypothalamic nucleus) (Fig. 1H-K).

\subsection{Behavioral assessment of nociception}

The rats were habituated to the experimental conditions by allowing them to spend $1-2 \mathrm{~h}$ daily in the laboratory during two to three days preceding any testing. For assessing nociception in unanaesthetized animals, radiant heat-induced latencies for withdrawing the tail and hindpaw (The Plantar Test Instrument Model 37370, Ugo Basile, Varese, Italy) were determined. Paw-withdrawal (PW) and tail-flick (TF) latencies were determined in separate sessions. In each drug treatment session, the withdrawal latency was assessed prior to drug administration and at various intervals 
Bicuculline

A

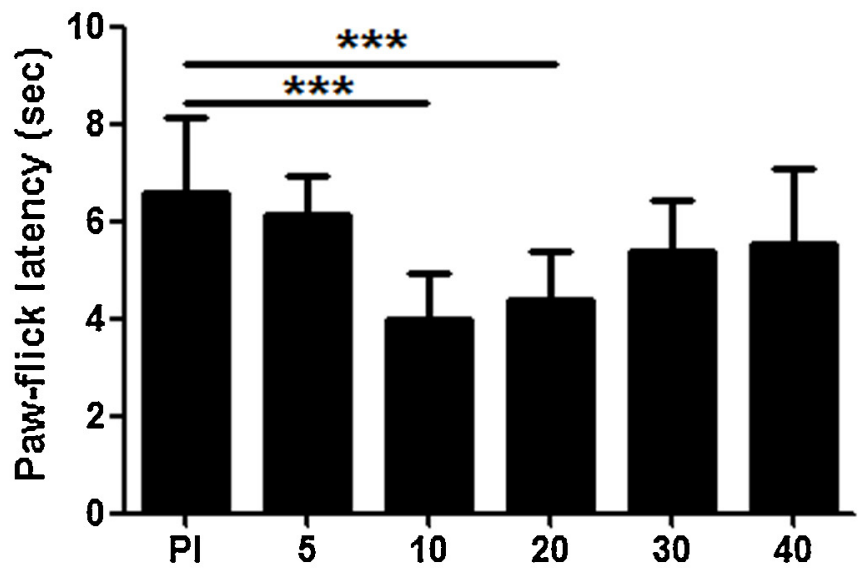

C

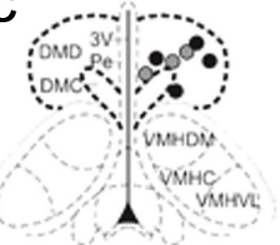

Bregma - $3.00 \mathrm{~mm}$

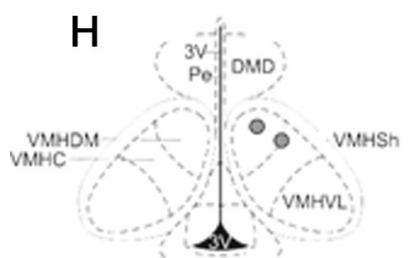

Bregma - $2.64 \mathrm{~mm}$

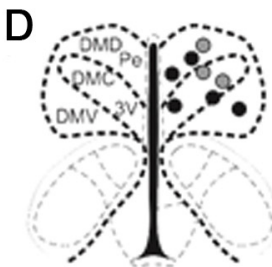

Bregma - $3.12 \mathrm{~mm}$

E

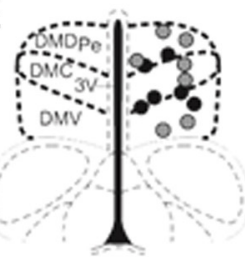

Bregma - $3.24 \mathrm{~mm}$

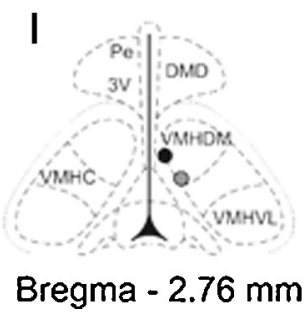

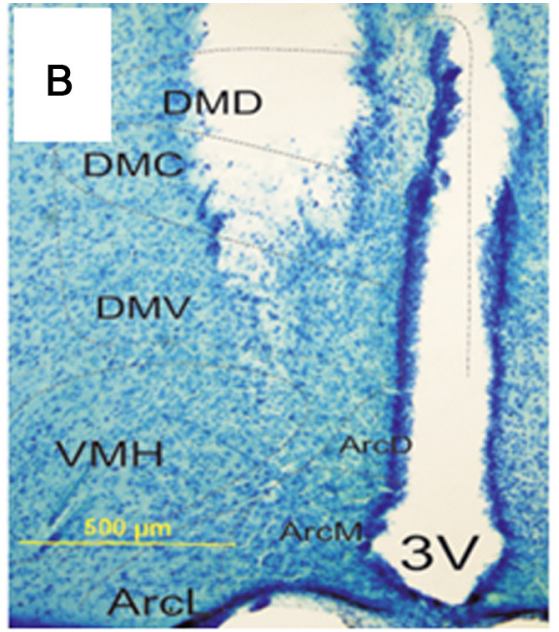

$\mathrm{F}$

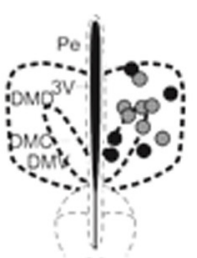

Bregma $-3.36 \mathrm{~mm}$

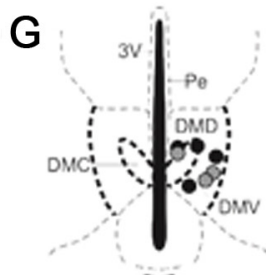

Bregma $-3.48 \mathrm{~mm}$
$\mathrm{J}$

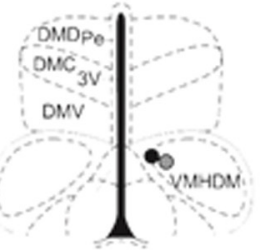

Bregma - $3.24 \mathrm{~mm}$
$\mathrm{K}$

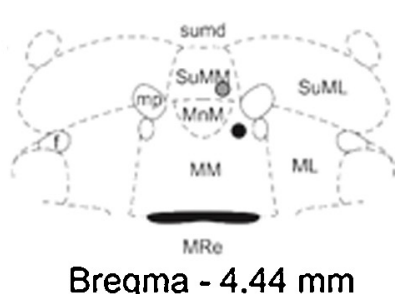

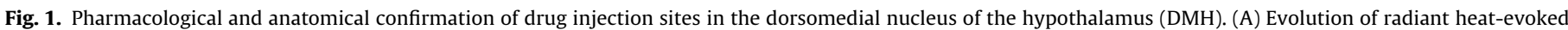

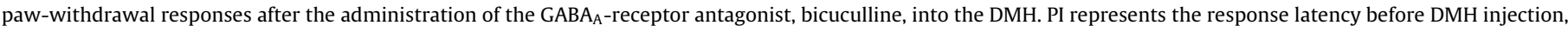

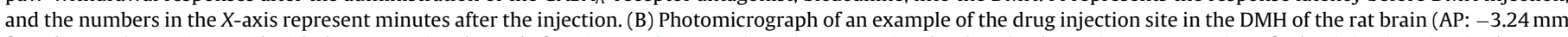

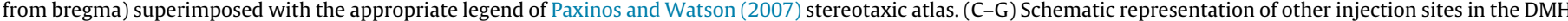

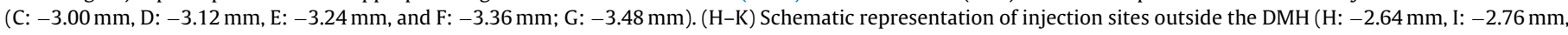

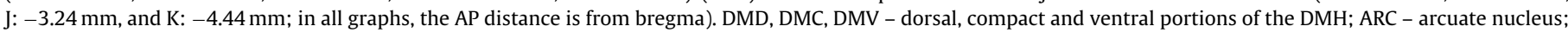

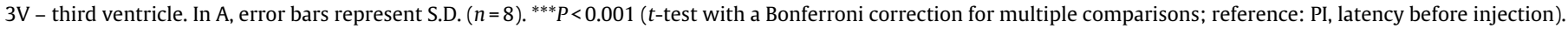

following the intracerebral injections. At each time point, the measurement was repeated (bilaterally for PW) twice at an interval of $1 \mathrm{~min}$ (except for GLU due to its fast effect) and the mean of these values was used in further calculations. Cut-off time was $15 \mathrm{~s}$.

\subsection{Course of the behavioral study}

The behavioral study started three weeks after the induction of arthritis and at least one week following insertion of the guide cannula in the DMH. Saline (SAL) was used in control injections of drugs, while NAIVE and SHAM animals were used as controls for ARTH animals. Paw-withdrawal latencies were measured $30 \mathrm{~s}$ after hypothalamic microinjection of GLU or 10 and $20 \mathrm{~min}$ after hypothalamic microinjection of LIDO, since the maximum effects by hypothalamic injections of these compounds have been obtained at these time points (Pinto-Ribeiro et al., 2008, 2011). The involvement of the RVM in mediating the descending pronociceptive drive from the DMH was assessed by pretreating the RVM with LIDO $15 \mathrm{~min}$ before administering GLU into the DMH. The interval between behavioral assessments of different drug treatment conditions in the same animal was at least two days. The order of testing different treatment conditions varied between the animals.

\subsection{Recording of neuronal responses in nociceptive RVM cells}

The electrophysiological recordings of the activity of RVM neurones followed a protocol described detail in Pinto-Ribeiro et al. (2008). Briefly, in anaesthetized animals a recording electrode was placed in the RVM (AP: $-1.92 \mathrm{~mm}$ from the interaural line, LM: 0.1-0.6 mm, and DV: $10.3 \mathrm{~mm}$ ) (Paxinos and Watson, 2007) using a standard stereotaxic frame. Single neurone activity was recorded extracellularly with a tungsten electrode (tip impedance 5-10 $\mathrm{M} \Omega$ at $1 \mathrm{kHz}$ ) and data sampling was performed using a CED Micro 1401 interface and Spike 2 software (Cambridge Electronic Design, Cambridge, UK).

For the characterization of RVM cells, we adapted the classification scheme developed by Fields et al., 1983 and Fields and Heinricher (1985). RVM neurones were classified as ON-like cells if their activity increased during peripheral noxious stimulation of the tail, OFF-like cells if their activity decreased during the noxious stimulation period and NEUTRAL-cells if no changes or only a 
A
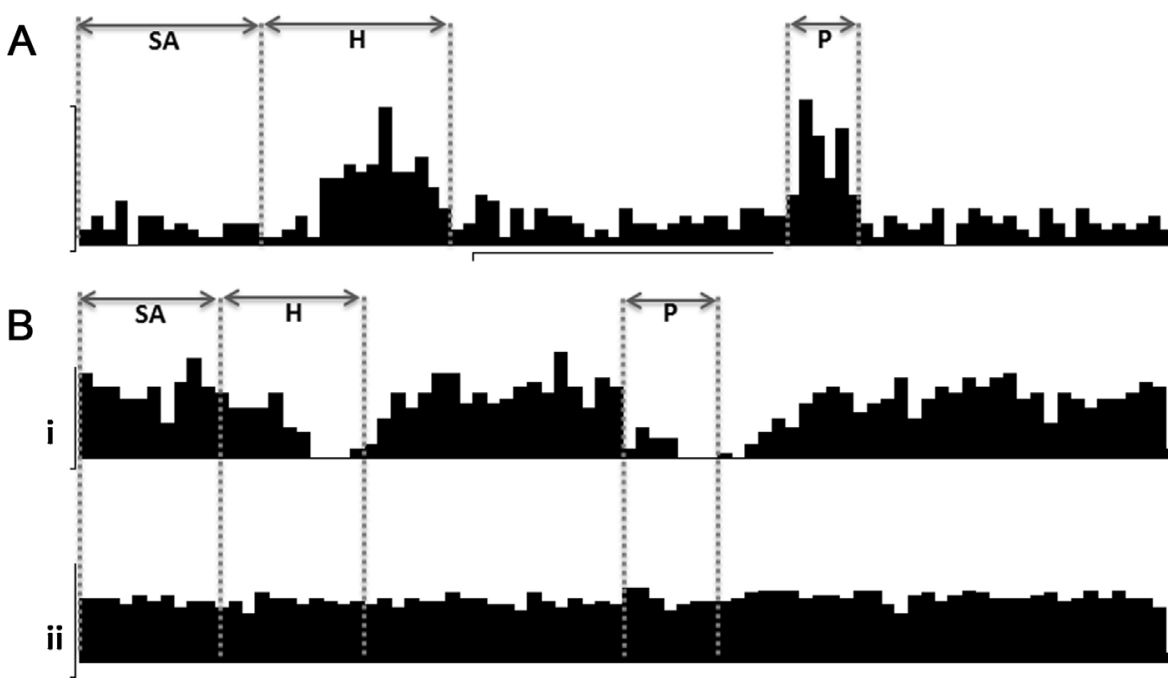

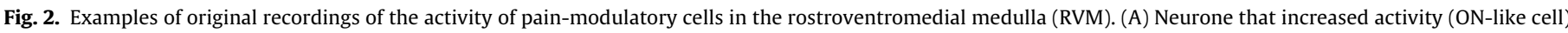

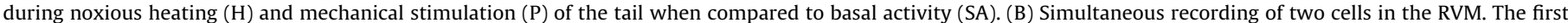

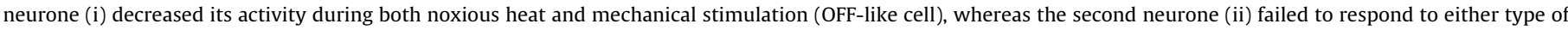

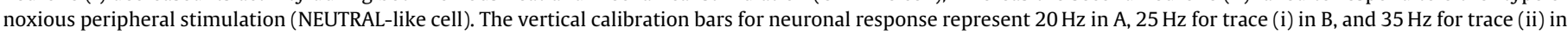
B. The horizontal calibration bar represents $25 \mathrm{~s}$ for graph A and $20 \mathrm{~s}$ for graph B.

negligible $(<10 \%)$ alteration in the discharge rates was observed (Fig. 2). It should be pointed out that in the present study the changes in the activity of RVM neurones were not directly correlated with withdrawal responses, unlike in the original classification scheme of Fields et al., 1983, but cell classification was solely based on the response to peripheral noxious stimulation of the tail, as in several earlier studies (Pertovaara et al., 2001; Miki et al., 2002; Pinto-Ribeiro et al., 2008; Sanoja et al., 2010; Sugino et al., 2012). As a result of this, RVM cells studied in the present study were designated as ON-like and OFF-like cells rather than ON- or OFF-cells. NEUTRAL-cells were not considered further in the present study.

The evaluation of the response of RVM cells to peripheral noxious stimulation consisted of the following assessments performed successively: (i) spontaneous activity (first $20 \mathrm{~s}$ of recording without any stimulation); (ii) response to heating of the tail ( $10 \mathrm{~s}$ or until a withdrawal response was observed), and (iii) response to pinching of the tail for $5 \mathrm{~s}$ with a surgical clamp that produced painful sensation when applied to the hand of the experimenter.

\subsection{Course of the electrophysiological study}

Electrophysiological recordings of RVM cells were performed three weeks following the induction of arthritis. Pharmacological manipulations started after the cell was classified as ON-like or OFF-like one and its spontaneous and noxious stimulation-evoked activity had been recorded. In a single session, one to four cells could be recorded simultaneously; the same cells were recorded throughout the whole session unless (1) the neurone stopped responding for more than an hour, in which case we would search for another recording site, or (2) the activity of the cell would not return to the same basal level as at the start of the recording in which case no more recordings were performed. Each session comprised the microinjection of SAL either GLU or LIDO, followed by a final injection of bicuculline. At the end of each recording session, an electrolytic lesion was made in the last recording site.

\subsection{Drugs}

Solutions for intracerebral drug injection in the DMH were prepared with sterilized saline $0.9 \%$ (Unither, Amiens, France; pH 7.2) except for LIDO, which was acquired as a solution. Each injection had a volume of $0.5 \mu \mathrm{L}$ and contained either $50 \mathrm{nmol}$ of GLU (Merck, Darmstadt, Germany) or LIDO 2\% (B. Braun Medical, Barcarena, Portugal). These doses were chosen according to previous studies (Pinto-Ribeiro et al., 2008; Ansah et al., 2009).

\subsection{Statistical analyses}

Behavioral data analysis was performed using an independentsamples $t$-test and one-way analysis of variance (ANOVA). The potential effect of drugs upon the spontaneous and noxiousevoked activity of pain modulatory-like cells of the RVM at specific timepoints was examined by using two-way analysis of variance (ANOVA). Post hoc tests were performed using $t$-test with a Bonferroni correction for multiple comparisons. $P<0.05$ was considered to represent a significant difference. Data in the results section is presented as mean \pm S.D.

\section{Results}

3.1. Induction of monoarthritis failed to alter pain-like behavior induced by noxious stimulation of non-inflamed skin areas

All animals in the ARTH group had monoarthritis of the knee as indicated by vocalization every time after flexion-extension movement of the affected but not the contralateral knee joint. In NAIVE or SHAM animals, flexion-extension movement of the knee joint failed to produce vocalizations. The baseline TF was not significantly different among the experimental groups $\left(F_{2,25}=3.340, P=0.5682\right.$; one-way-ANOVA) (Fig. 3A). Baseline PW latencies were not significantly different between the left and right hindpaws in the $\operatorname{NAIVE}\left(t_{(0,636)}=0.53\right.$; paired $t$-test $)$, SHAM $\left(t_{(0,491)}=0.64\right)$ or ARTH $\left(t_{(2,107)}=0.07\right)$ groups. Therefore, the mean PW data for left and right hindpaws in each experimental group were used in further calculations. The baseline PW was significantly different among the experimental groups $\left(F_{2,28}=4.279, P<0.0247\right.$; one-way-ANOVA). The ARTH group had the longest PW latency $(7.03 \pm 0.13 \mathrm{~s})$ in the baseline condition, while the PW latencies were in the same range in NAIVE (6.14 $\pm 0.26 \mathrm{~s})$ and SHAM ( $5.94 \pm 0.08 \mathrm{~s})$ groups (Fig. 3B). One NAIVE animal and one SHAM animal were excluded from the experiment for severe weight loss during the first week of 

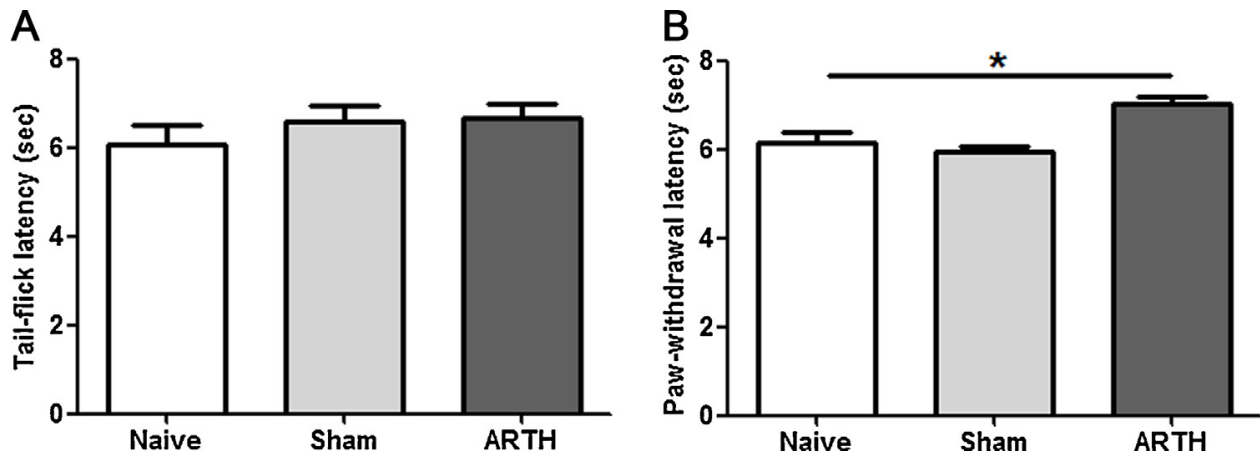

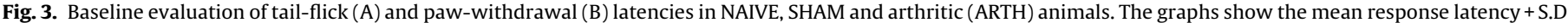
${ }^{*} P<0.05$ (reference in $A$ and $B$ : the NAIVE group).

behavioral assessment. We were unable to ascertain a probable cause for the weight loss, although we performed necropsis.

\subsection{Effect of DMH manipulation upon nociceptive behavior}

\subsubsection{DMH activation increased nociceptive behavior in control} but not arthritic animals

TF and PW latencies were assessed following GLU administration in the DMH to study phasic modulation of pain behavior by activation of the DMH. GLU in the DMH produced a significant decrease of the TF latency (main effect of GLU: $F_{1,56}=33.37$, $P<0.0001$ ). The TF latency was significantly different among the experimental groups (main effect of the experimental group: $F_{2,56}=4.915, P=0.01$ ) and the difference in the TF latency among experimental groups varied with the drug treatment (interaction between experimental group and drug treatment: $F_{2,56}=4.928$,
$P=0.0107$ ). Post hoc testing indicated that the TF latency was decreased by GLU in the DMH only in the NAIVE and SHAM groups but not in the ARTH group (Fig. 4A).

Identically, GLU in the DMH induced a significant decrease of the PW latency (main effect of GLU: $F_{1,59}=15.59, P=0.0002$ ). PW latency was significantly different among the experimental groups (main effect of the experimental group: $F_{2,59}=5.555, P=0.0062$ ). Again, post hoc testing showed that the PW latency was decreased by GLU in the DMH only in the NAIVE and SHAM groups but not in the ARTH group (Fig. 4B).

\subsubsection{DMH inhibition decreased nociceptive behavior in control} but not arthritic animals

Lido in the DMH significantly increased TF latency (main effect of LIDO: $F_{1,65}=24.94, P<0.0001$ ) although this effect was not significantly different among experimental groups (main effect of the
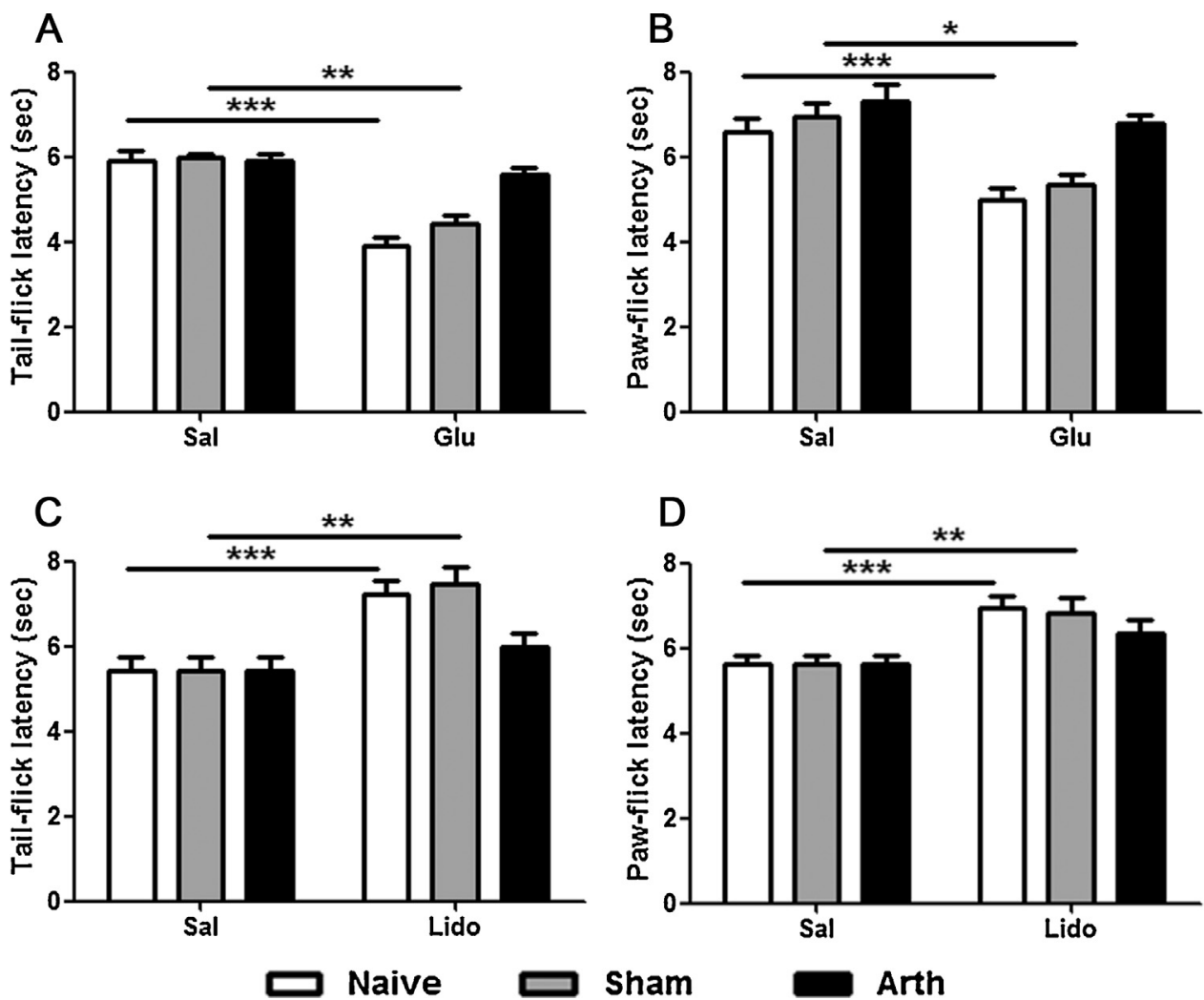

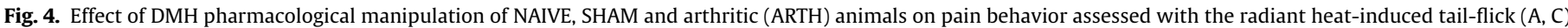

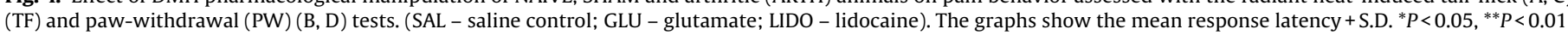
${ }^{* * *} P<0.001$ (reference in $\mathrm{A}$ and $\mathrm{B}$ : the corresponding SAL group; reference in C and D: the corresponding PI group). 


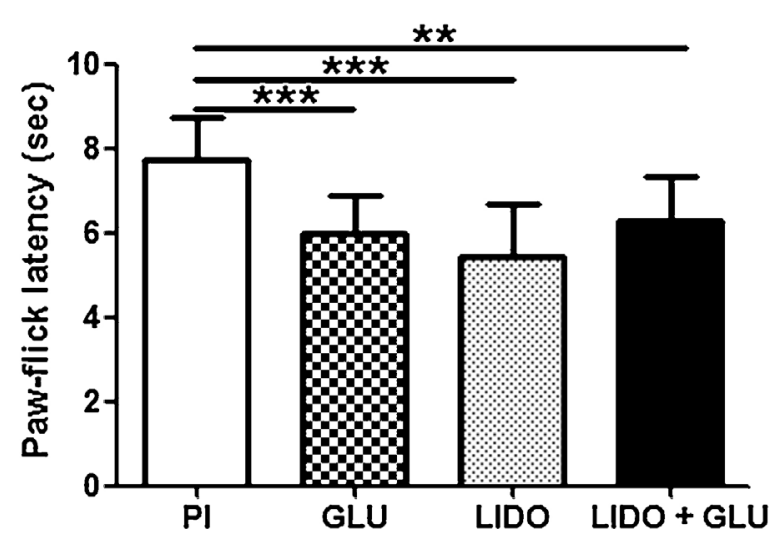

Fig. 5. Comparison of paw-withdrawal (PW) latencies in NAIVE animals after (i) microinjection of glutamate (GLU) into the DMH, (ii) lidocaine (LIDO) into the rostroventromedial medulla (RVM) and (iii) lidocaine into the RVM followed by glutamate into the DMH (20 min later). Note that all the pharmacological treatments significantly decreased PW latencies when compared to preinjection (PI) baseline responses, but no differences were observed between treatments. Graphs show mean latency + S.D. $(n=14) .{ }^{* *} P<0.01,{ }^{* * *} P<0.001$ ( $t$-test with a Bonferroni correction for multiple comparisons; reference: the PI group).

experimental group: $\left.F_{2,56}=2.451, P=0.0941\right)$. Post hoc testing indicated that the TF latency was increased by LIDO in the DMH only in the NAIVE and SHAM groups but not in the ARTH group (Fig. 4C).

Identically, LIDO in the DMH induced a significant increase in PW latency (main effect of LIDO: $F_{2,64}=27.55, P<0.0001$ ) although this effect was not significantly different among experimental groups (main effect of the experimental group: $F_{1,64}=0.8238$, $P=0.4434$ ). Again, post hoc testing showed that the PW latency was increased by LIDO in the DMH only in the NAIVE and SHAM groups but not in the ARTH group (Fig. 4D).

\subsection{Increased nociceptive behavior by the DMH is mediated by the RVM}

Next, we assessed whether the RVM is a possible downstream medullary relay for the $\mathrm{DMH}$-induced pronociceptive action in normal animals. Therefore, we determined PW latency following administration of GLU into the DMH of SHAM animals with a LIDOinduced block of the RVM. For comparison, PW latency was also measured following administration of GLU into the DMH without a concomitant block of the RVM, or following a LIDO induced block of the RVM without a concomitant administration of GLU into the DMH. All three treatments (the activation of the DMH by GLU, the inhibition of the RVM by LIDO, or the concomitant inhibition of the RVM with LIDO and activation of the DMH by GLU) resulted in a decrease in PW latency that was significantly different from the pre-treatment baseline $\left(F_{3,55}=13.51, P<0.0001\right)$. Post hoc tests indicated that there were no differences in the post-injection PW latencies among the three treatment groups (Fig. 5).

\subsection{Effect of DMH manipulation upon the activity of RVM cells}

\subsubsection{The sample of RVM cells studied}

A total of 102 RVM cells of SHAM animals were analyzed [69 ON-like cells: 39 ON-like cells tested with mechanical stimuli (MS; noxious pinch of the tail) and $30 \mathrm{ON}$-like cells tested with thermal stimuli (TS; noxious heating of the tail); 33 OFF-like cells: 19 OFFlike cells tested with MS and 14 OFF-like cells tested with TS], while the number of RVM cells analyzed in ARTH animals was 253 [137 ON-like cells: 71 ON-like cells tested with MS and 66 ON-like cells tested with TS; 116 OFF-like cells: 65 OFF-like cells tested with MS and 51 OFF-like cells tested with TS].
3.4.2. DMH manipulation altered the spontaneous activity of RVM cells in a pronociceptive fashion, an effect that was absent in arthritic animals

Microinjection of drugs into the DMH altered significantly the spontaneous activity of ON-like RVM cells $\left(F_{2,159}=5.307\right.$, $P=0.0059)$. This effect varied with the experimental group $\left(F_{2,159}=11.18, P<0.0001\right)$. Post hoc testing indicated that GLU significantly increased ON-like cell spontaneous activity in the SHAM group while LIDO increased ON-like cell spontaneous activity in the ARTH group (Fig. 6A).

Similarly, spontaneous activity of OFF-like RVM cells was altered by drug treatments of the DMH $\left(F_{2,90}=10.38, P<0.0001\right)$, an effect that also varied with the experimental group $\left(F_{2,90}=12.37\right.$, $P<0.0001)$. Post hoc testing showed that GLU significantly decreased OFF-like cell spontaneous activity only in the SHAM group whereas LIDO had no effect on spontaneous activity of OFFlike cells, independent of the experimental group (Fig. 6B).

\subsubsection{DMH manipulation altered the noxious stimulation-evoked responses of RVM cells, an effect that was absent in arthritic animals}

Drug treatments of the DMH had a significant main effect on the magnitude of the heat stimulation-evoked and or mechanical stimulation-evoked response in RVM ON-like cells (HEAT: $F_{2,77}=14.66, P<0.0001$ and MECHANICAL: $\left.F_{2,95}=26.18, P<0.0001\right)$, which varied between experimental groups (HEAT: $F_{2,77}=13.35$, $P<0.0001$ and MECHANICAL: $\left.F_{2,95}=26.22, P<0.0001\right)$. Post hoc testing showed that GLU increased and LIDO decreased ON-like cell responses to heat in SHAM animals whereas no effect was observed in the ARTH group (Fig. 7A, C). Concomitantly, drug treatment of the DMH also significantly altered the noxious stimulationevoked activity of OFF-like RVM cells (HEAT: $F_{2,47}=22.34, P<0.0001$ and MECHANICAL: $\left.F_{2,66}=43.52, P<0.0001\right)$. Again, the effect of drug treatment on the peripheral stimulation-evoked responses of OFF-like cells varied with experimental group (HEAT: $F_{2,47}=18.13$, $P<0.0001$ and MECHANICAL: $\left.F_{2,66}=47.03, P<0.0001\right)$. Although no effect upon ARTH animals was observed, in the SHAM group GLU decreased and LIDO increased RVM OFF-like cell responses during heat stimulation (Fig. 7B, D).

\section{Discussion}

In line with earlier results (Martenson et al., 2009), our study indicates that the $\mathrm{DMH}$ has a pronociceptive role, enhancing acute pain-like behavior of rodents in a phasic and tonic fashion. Moreover, the DMH-induced facilitation of pain behavior was accompanied by the induction of pronociceptive changes in the activity of pain-modulatory RVM ON-like and OFF-like cells of healthy control animals as in the study of Martenson et al. (2009). Importantly, in contrast to other supraspinal areas that enhance nociception in acute and chronic pain conditions (Almeida et al., 1996, 1999; Pinto et al., 2008; Sotgiu et al., 2008; Martins et al., 2010), the novel finding of the present study was that the DMHinduced facilitation of pain-like behavior was absent in arthritic animals. Concomitantly, the pronociceptive effect of the DMH upon pain-regulating RVM cells was also absent in arthritic animals. The present data suggests that the pronociceptive role of the DMH is restricted to acute pain but is lost or altered in prolonged pain conditions (like arthritis).

\subsection{Role of the DMH in acute pain modulation}

In accordance to a potential role in pain facilitation, the DMH activation by GLU and inhibition by LIDO clearly demonstrated a facilitatory role for this nucleus in acute pain behavior. These results are in line with earlier data showing that disinhibition of the $\mathrm{DMH}$ 


\section{Spontaneous activity of RVM ON- and OFF-like cells}
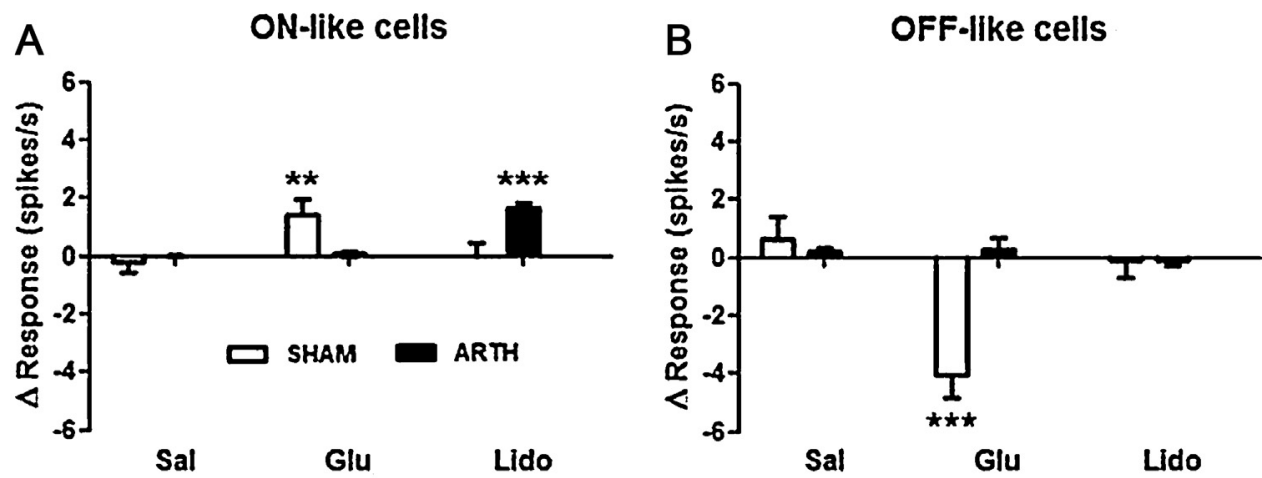

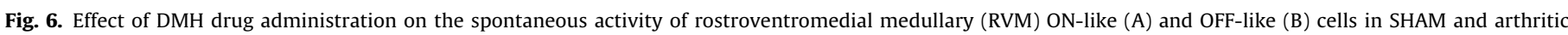

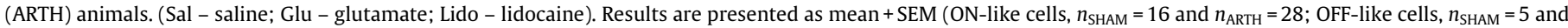
$\left.n_{\text {ARTH }}=21\right)$. ${ }^{* * *} P<0.001$.

by bicuculline methiodide, a $\mathrm{GABA}_{\mathrm{A}}$ receptor antagonist, induces behavioral hyperalgesia (Martenson et al., 2009), a finding that was replicated also in the present study.

In an attempt to integrate $\mathrm{DMH}$ and brainstem activity, Zaretskaia et al. (2008) evaluated the level of c-Fos expression in the rat brain after disinhibition of the DMH. Among central nervous system regions in which DMH disinhibition enhanced c-Fos expression was the RVM (Zaretskaia et al., 2008). This is noteworthy since the RVM, together with some other brainstem areas (Lima and Almeida, 2002; Kincaid et al., 2005; Waters and Lumb, 2008; Marques-Lopes et al., 2010), is able to facilitate nociception. Additionally, electrophysiological studies have already demonstrated a pronociceptive action of the DMH on the RVM (Heinricher et al., 2009). In the present study, GLU in the DMH of healthy control animals induced pronociceptive effects both on noxious stimulusevoked responses and spontaneous activities of RVM ON-like and OFF-like cells. Thus, the GLU-induced phasic pronociceptive effect in control animals can be explained by a direct descending action

\section{Noxious-evoked responses of RVM ON- and OFF-like cells}

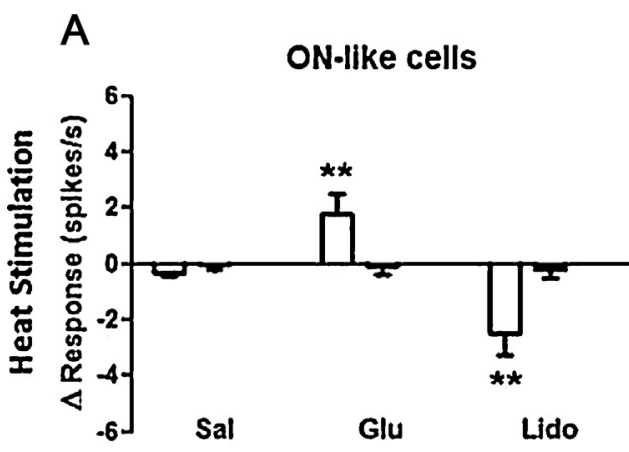

C

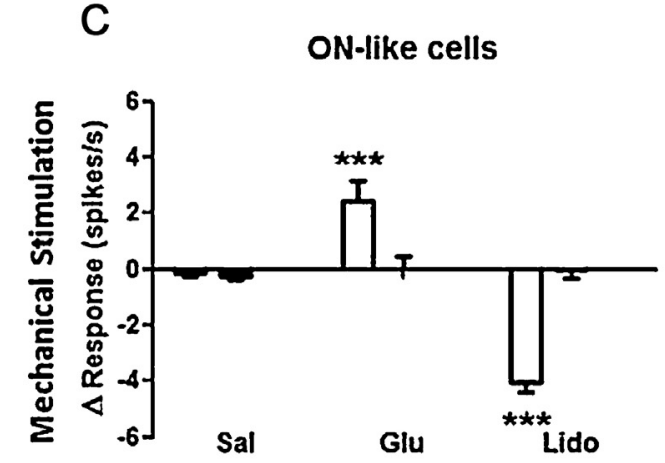

B

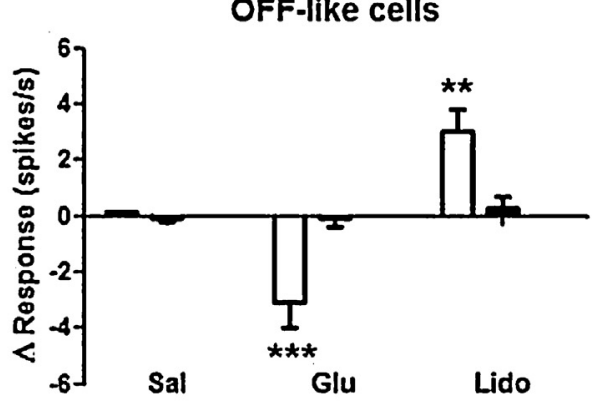

D

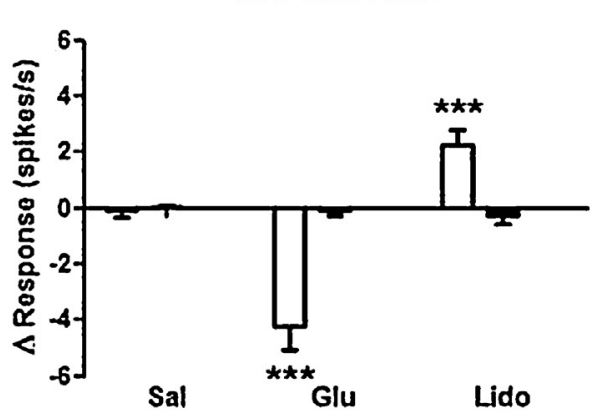

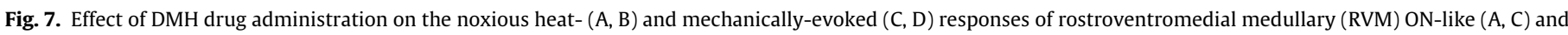

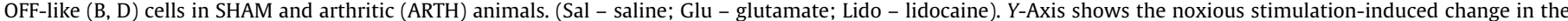

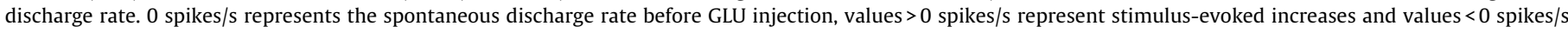

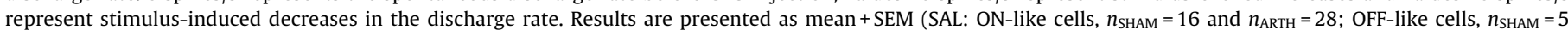

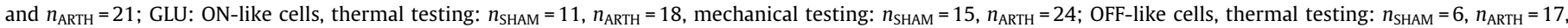

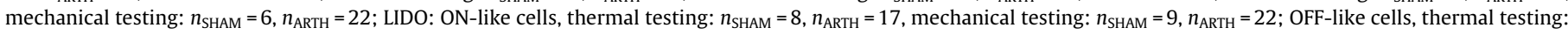
$n_{\mathrm{SHAM}}=6, n_{\mathrm{ARTH}}=8$, mechanical testing: $\left.n_{\mathrm{SHAM}}=11, n_{\mathrm{ARTH}}=11\right) .{ }^{*} P<0.05,{ }^{* *} P<0.01,{ }^{* * *} P<0.001$. 
of the DMH on RVM cells that was followed by enhanced spinal nociceptive transmission. The hypothesis that the phasic pronociceptive effect induced by activation of the DMH in control animals was mediated by the RVM rather than other areas of the brain is partly supported by the finding that GLU activation of the DMH after blocking the RVM failed to result in an additional increase in the descending pronociceptive effect (blocking the RVM is already pronociceptive by itself).

LIDO treatment was used to study tonic control of nociception by the DMH. Blocking the DMH with LIDO in control animals failed to change the spontaneous activity of RVM ON-like or OFF-like cells while it reduced the magnitude of the noxious stimulationevoked responses of RVM ON- and OFF-like cells. The reduction in stimulus-evoked responses of RVM cells following LIDO treatment of the DMH was accompanied by a reduction in stimulus-evoked pain behavior. This finding suggests that, in control animals, the tonic descending effect of the DMH on RVM cells and pain behavior is presynaptic rather than postsynaptic to the RVM. This hypothesis is in line with the work of Yoshimura and Hidemasa (2006) that showed that evoked activity of spinal neurones was under pre-synaptic control of descending noradrenergic projections.

Several authors have reported that the RVM contributes to the descending modulation of nociception in chronic pain conditions (e.g., Pertovaara et al., 1996; Vera-Portocarrero et al., 2011; Ambriz-Tututi et al., 2011; Mase et al., 2011). Spinally-projecting RVM neurones, known to be involved in this process (Urban et al., 1999; Porreca et al., 2002) include those expressing $G_{A B A}$ (Heinricher and Kaplan, 1991) receptors. Interestingly, the existence of a GABAergic output from the DMH (Acosta-Galvan et al., 2011) and of DMH neurons projecting to the RVM (Thompson et al., 1996) have already been demonstrated. Therefore, it is conceivable that the acute hyperalgesia observed in control animals following GLU administration into the DMH might result from activation of DMH GABAergic projecting neurones that target spinally-projecting RVM neurones. Further studies involving pharmacological manipulations of the spinal cord and RVM during DMH activation should clarify the neurotransmitter mechanisms of this hypothalamic-medullary interaction.

\subsection{Role of the DMH in arthritis}

The failure to influence pain behavior by activation or inhibition of the DMH in arthritic animals, which was paralleled by a similar failure in altering the activity of RVM nociceptive neurones was intriguing. An increase in the descending pronociceptive drive originating in the $\mathrm{DMH}$ would have been expected as chronic inflammatory disorders lead to changes toward pronociception in the balance of descending modulation (Miki et al., 2002; Dubner, 2005; Woolf and Salter, 2006; Lagraize et al., 2010). On the other hand, chronic inflammation also results in an increased descending antinociceptive drive (Cervero et al., 1991; Danziger et al., 2001) in an attempt to counteract increased pain. It is conceivable that the loss of descending pain-facilitatory effect by the DMH following development of arthritis might also result from this compensatory phenomenon.

An aspect to take into consideration is that we did not evaluate primary hyperalgesia (knee). While it has been demonstrated (Urban et al., 1999) that hyperalgesia resulting from an injury is not restricted to the lesioned region, some authors argue that descending pain modulatory pathways differently modulate peripheral inputs according to their location (Vanegas and Schaible, 2004). Indeed, Urban and Gebhart (1999) showed the occurrence of descending facilitation of nociceptive inputs originating from healthy areas while inputs arising from the injury site were inhibited. Interestingly, RVM lesions would prevent the descending facilitatory drive of inputs originating from healthy areas surrounding the lesion site but not from the lesion site (Urban and Gebhart, 1999). In this context, the loss of the DMH-induced pain facilitation in arthritic animals may result from the remodeling of the pathways signaling and modulating nociception in prolonged chronic conditions, both at the spinal and supraspinal levels. Whether this "shut-down" of the DMH is associated with changes in the neurochemistry of the hypothalamus or the brainstem remains to be determined.

At the hypothalamic level, we have previously demonstrated that after the induction of arthritis, the paraventricular nucleus of the hypothalamus (PVN) tonically enhanced the firing rate of antinociceptive RVM OFF-like cells, in parallel to an antinociceptive behavioral effect (Pinto-Ribeiro et al., 2008). Similarly, it is possible that in the present study arthritis enhanced the tonic inhibitory drive from the hypothalamus, either locally by inhibiting pronociceptive DMH activity, at the brainstem level by inhibiting pronociceptive activity of RVM cells, or/and spinally by inhibiting nociceptive transmission.

Interestingly, the increase in the spontaneous activity of pronociceptive RVM ON-like cells after blocking the DMH with LIDO in ARTH animals was an exception to the general data, suggesting the existence of a tonic inhibitory descending drive in prolonged arthritis. This result not only further supports the hypothesis of the existence of hypothalamic plasticity in chronic inflammation but also implicates that the DMH may contribute to a tonically-mediated inhibition of pronociceptive RVM cells after the development of arthritis. This tonic inhibition of the RVM ON-like cell activity by the DMH in arthritic animals, however, did not produce a net change in the balance of descending pain-facilitatory and -inhibitory controls as indicated by the failure to produce a change in pain behavior of arthritic animals after blocking the DMH. This is probably due to the development of a strong pronociceptive descending drive in the chronic phase of arthritis (Danziger et al., 2001).

\section{Conclusions}

The DMH plays a role in acute pain facilitation in normal animals, and this effect is associated with pronociceptive alterations in the activity of pain-regulating RVM cells. However, in arthritic animals, the DMH-induced pronociception is lost, probably due to remodeling of the hypothalamic circuits mediating nociception. Additionally, a tonic descending inhibitory drive originating in the DMH was present in arthritic but not control animals, further supporting the hypothesis of remodeling of hypothalamic circuits in chronic inflammatory conditions.

\section{Conflict of interest}

The authors declare that there are no conflicts of interest.

\section{Acknowledgements}

This study was supported by grants from the Portuguese Science Foundation (FCT) Project $n^{\circ}$ PTDC/SAU-NEU/108557/2008, FEDER-COMPETE and Grünenthal Foundation, and the Academy of Finland. Diana Amorim and Ana David-Pereira were supported by the FCT grants SFRH/BD/71219/2010 and SFRH/BD/90374/2012, respectively.

\section{References}

Ambriz-Tututi, M., Cruz, S.L., Urquiza-Marin, H., Granados-Soto, V., 2011. Pharmacol. Biochem. Behav. 98, 417-424.

Acosta-Galvan, G., Yi, C.X., van der Vliet, J., Jhamandas, J.H., Panula, P., AngelesCastellanos, M., Del Carmen Basualdo, M., Escobar, C., Buijs, R.M., 2011. 
Interaction between hypothalamic dorsomedial nucleus and the suprachiasmatic nucleus determines intensity of food anticipatory behavior. Proc. Natl. Acad. Sci. U. S. A. 108, 5813-5818.

Almeida, A., Størkson, R., Lima, D., Hole, K., Tjølsen, A., 1999. The medullary dorsal reticular nucleus facilitates pain behaviour induced by formalin in the rat. Eur. J. Neurosci. 11, 110-122.

Almeida, A., Tjølsen, A., Lima, D., Coimbra, A., Hole, K., 1996. The medullary dorsal reticular nucleus facilitates acute nociception in the rat. Brain Res. Bull. 39, 7-15.

Ansah, O.B., Gonçalves, L., Almeida, A., Pertovaara, A., 2009. Enhanced pronociception by amygdaloid group I metabotropic glutamate receptors in nerve-injured animals. Exp. Neurol. 216, 66-74.

Ansah, O.B., Pertovaara, A., 2007. Peripheral suppression of arthritic pain by intraarticular fadolmidine, an $\alpha_{2}$-adrenoceptor agonist, in the rat. Anesth. Analg. 105, 245-250.

Burgess, S.E., Gardell, L.R., Ossipov, M.H., Malan Jr., T.P., Vanderah, T.W., Lai, J., Porreca, F., 2002. Time-dependent descending facilitation from the rostral ventromedial medulla maintains, but does not initiate, neuropathic pain. J. Neurosci. 22, 5129-5136.

Cervero, F., Schaible, H.G., Schmidt, R.F., 1991. Tonic descending inhibition of spinal cord neurones driven by joint afferents in normal cats and in cats with an inflamed knee joint. Exp. Brain Res. 83, 675-678.

Danziger, N., Weil-Fugazza, J., Le Bars, D., Bouhassira, D., 2001. Stage-dependent changes in the modulation of spinal nociceptive neuronal activity during the course of inflammation. Eur. J. Neurosci. 13, 230-240.

Dubner, R., 2005. Plasticity in central nociceptive pathways. In: Merskey, H., Loeser, J.D., Dubner, R. (Eds.), The Paths of Pain. IASP Press, Seattle, pp. 101-116.

Fields, H.L., Bry, J., Hentall, I., Zorman, G., 1983. The activity of neurons in the rostral medulla of the rat during withdrawal from noxious heat. J. Neurosci. 3 , 2545-2552.

Fields, H.L., Heinricher, M.M., 1985. Anatomy and physiology of a nociceptive modulatory system. Philos. Trans. R. Soc. Lond. B: Biol. Sci. 308, 361-374.

Gozariu, M., Bouhassira, D., Willer, J.C., Le Bars, D., 1998. The influence of temporal summation on a C-fibre reflex in the rat: effects of lesions in the rostral ventromedial medulla (RVM). Brain Res. 792, 168-172.

Heinricher, M.M., Kaplan, H.J., 1991. GABA-mediated inhibition in rostral ventromedial medulla: role in nociceptive modulation in the lightly anesthetized rat. Pain 47, 105-113.

Heinricher, M.M., Tavares, I., Leith, J.L., Lumb, B.M., 2009. Descending control of nociception: specificity, recruitment and plasticity. Brain Res. Rev. 60, 214-225.

Herrero, J.F., Cervero, F., 1996. Supraspinal influences on the facilitation of rat nociceptive reflexes induced by carrageenan monoarthritis. Neurosci. Lett. 209, $21-24$

Kincaid, W., Neubert, M.J., Xu, M., Kim, C.J., Heinricher, M.M., 2005. Role for medullary pain facilitating neurons in secondary thermal hyperalgesia. J. Neurophysiol. 95, $33-41$

Lagraize, S.C., Guo, W., Yang, K., Wei, F., Ren, K., Dubner, R., 2010. Spinal cord mechanisms mediating behavioral hyperalgesia induced by neurokinin-1 tachykinin receptor activation in the rostral ventromedial medulla. Neuroscience 171, 1341-1356.

Latremoliere, A., Woolf, C.J., 2009. Central sensitization: a generator of pain hypersensitivity by central neural plasticity. J. Pain 10, 895-926.

Lima, D., Almeida, A., 2002. The medullary dorsal reticular nucleus as a pronociceptive centre of the pain control system. Prog. Neurobiol. 66, 81-108.

Marques-Lopes, J., Pinho, D., Albino-Teixeira, A., Tavares, I., 2010. The hyperalgesic effects induced by the injection of angiotensin II into the caudal ventrolateral medulla are mediated by the pontine A5 noradrenergic cell group. Brain Res. $1325,41-52$

Martenson, M.E., Cetas, J.S., Heinricher, M.M., 2009. A possible neural basis for stressinduced hyperalgesia. Pain 142, 236-244

Martins, I., Costa-Araújo, S., Fadel, J., Wilson, S.P., Lima, D., Tavares, I., 2010. Reversal of neuropathic pain by HSV-1-mediated decrease of noradrenaline in a pain facilitatory area of the brain. Pain 151, 137-145.

Mase, H., Sakai, A., Sakamoto, A., Suzuki, H., 2011. A subset of $\mu$-opioid receptor-expressing cells in the rostral ventromedial medulla contribute to thermal hyperalgesia in experimental neuropathic pain. Neurosci. Res. 70, 35-43.

Miki, K., Zhou, Q.Q., Guo, W., Guan, Y., Terayama, R., Dubner, R., Ren, K., 2002. Changes in gene expression and neuronal phenotype in brain stem pain modulatory circuitry after inflammation. J. Neurophysiol. 87, 750-760.

Myers, R.D., 1966. Injection of solutions into cerebral tissue: relation between volume and diffusion. Physiol. Behav. 1, 171-174.
Ossipov, M.H., Lai, J., King, T., Vanderah, T.W., Porreca, F., 2005. Underlying mechanisms of pronociceptive consequences of prolonged morphine exposure. Biopolymers 80, 319-324.

Ossipov, M.H., Dussor, G.O., Porreca, F., 2010. Central modulation of pain. J. Clin. Invest. 120, 3779-3787.

Paxinos, G., Watson, C., 2007. The Rat Brain in Stereotaxic Coordinates, 6th ed. Academic Press, Sydney.

Pertovaara, A., Wei, H., Hämäläinen, M.M., 1996. Lidocaine in the rostroventromedia medulla and the periaqueductal gray attenuates allodynia in neuropathic rats. Neurosci. Lett. 218, 127-130.

Pertovaara, A., Keski-Vakkuri, U., Kalmari, J., Wei, H., Panula, P., 2001. Response properties of neurons in the rostroventromedial medulla of neuropathic rats: attempted modulation of responses by [1DMe]NPYF, a neuropeptide FF analogue. Neuroscience 105, 457-468.

Pinto, M., Castro, A.R., Tshudy, F., Wilson, S.P., Lima, D., Tavares, I., 2008. Opioids modulate pain facilitation from the dorsal reticular nucleus. Mol. Cell. Neurosci. 39, 508-518.

Pinto-Ribeiro, F., Ansah, O.B., Almeida, A., Pertovaara, A., 2008. Influence of arthritis on descending modulation of nociception from the paraventricular nucleus of the hypothalamus. Brain Res. 1197, 63-75.

Pinto-Ribeiro, F., Ansah, O.B., Almeida, A., Pertovaara, A., 2011. Response properties of nociceptive neurons in the caudal ventrolateral medulla (CVLM) in monoarthritic and healthy control rats: modulation of responses by the paraventricular nucleus of the hypothalamus (PVN). Brain Res. Bull. 86, 82-90.

Porreca, F., Ossipov, M.H., Gebhart, G.F., 2002. Chronic pain and medullary descending facilitation. Trends Neurosci. 25, 319-325.

Radhakrishnan, R., Moore, S.A., Sluka, K.A., 2003. Unilateral carrageenan injection into muscle or joint induces chronic bilateral hyperalgesia in rats. Pain 104 567-577.

Ren, K., Dubner, R., 1996. Enhanced descending modulation of nociception in rats with persistent hindpaw inflammation. J. Neurophysiol. 76, 3025-3037.

Sandkühler, J., 2009. Models and mechanisms of hyperalgesia and allodynia. Physiol Rev. 89, 707-758

Sanoja, R., Tortorici, V., Fernandez, C., Price, T.J., Cervero, F., 2010. Role of RVM neurons in capsaicin-evoked visceral nociception and referred hyperalgesia. Eur. J. Pain 14 (120), e1-e9.

Schaible, H.G., Ebersberger, A., Von Banchet, G.S., 2002. Mechanisms of pain in arthritis. Ann. N. Y. Acad. Sci. 966, 343-354.

Sotgiu, M.L., Valente, M., Storchi, R., Caramenti, G., Mario Biella, G.E., 2008. Contribution by DRt descending facilitatory pathways to maintenance of spinal neuron sensitization in rats. Brain Res. 1188, 69-75.

Sugino, S., Namiki, A., Yamakage, M., 2012. Genetic differences in response properties of rostral ventromedial medulla neurons to the $\mu$-opioid receptor agonist DAMGO in mouse inbred strains. Neurosci. Lett. 517, 107-112.

Thompson, R.H., Canteras, N.S., Swanson, L.W., 1996. Organization of projections from the dorsomedial nucleus of the hypothalamus: a PHA-L study in the rat. J. Comp. Neurol. 376, 143-173.

Urban, M.O., Gebhart, G.F., 1999. Supraspinal contributions to hyperalgesia. Proc Natl. Acad. Sci. U. S. A. 96, 7687-7692.

Urban, M.O., Zahn, P.K., Gebhart, G.F., 1999. Descending facilitatory influences from the rostral medial medulla mediate secondary, but not primary hyperalgesia in the rat. Neuroscience $90,349-352$.

Vanegas, H., 2004. To the descending pain-control system in rats, inflammationinduced primary and secondary hyperalgesia are two different things. Neurosci. Lett. 361, 225-228.

Vanegas, H., Schaible, H.G., 2004. Descending control of persistent pain: inhibitory or facilitatory? Brain Res. Brain Res. Rev. 46, 295-309.

Vera-Portocarrero, L.P., Ossipov, M.H., Lai, J., King, T., Porreca, F., 2011. Descending facilitatory pathways from the rostroventromedial medulla mediate naloxoneprecipitated withdrawal in morphine-dependent rats. J. Pain 12, 667-676.

Waters, A.J., Lumb, B.M., 2008. Descending control of spinal nociception from the periaqueductal grey distinguishes between neurons with and without C-fibre inputs. Pain 134, 32-40.

Woolf, C.J., Salter, M.W., 2006. Plasticity and pain: role of the dorsal horn. In: McMahon, S.B., Koltzenburg, M. (Eds.), Wall and Melzack's Textbook of Pain. , 5th ed. Elsevier, Churchill, Livingstone, pp. 91-105.

Yoshimura, M., Hidemasa, F., 2006. Mechanisms for the anti-nociceptive actions of the descending noradrenergic and serotonergic systems in the spinal cord. J. Pharmacol. Sci. 101, 107-117

Zaretskaia, M.V., Zaretsky, D.V., Sarkar, S., Shekhar, A., DiMicco, J.A., 2008. Induction of Fos-immunoreactivity in the rat brain following disinhibition of the dorsomedial hypothalamus. Brain Res. 1200, 39-50. 